\title{
Analytical solution of an Eulerian dilute particle-laden flow problem
}

\author{
Yahia M. Fouda ${ }^{1}$
}

(c) Springer Nature Switzerland AG 2019

\begin{abstract}
Dilute particle-laden flows are encountered in various natural processes and manmade applications. To reduce the computational resources used to simulate such flows, the particle phase could be formulated using the Eulerian approach, resulting in a continuum hydrodynamic model. The aim of this paper is to present the analytical solution of a steady two-dimensional flow problem using that model. The dispersed solid particles, considered in this problem, are immersed in a uniform fluid flow field. The solid phase is coupled to the fluid using a linear Stokes-drag, which is valid for low slip velocities. The general solution of the solid phase velocity field is obtained by solving its quasi-linear hyperbolic momentum equations using the method of characteristics. For the case of a uniform inlet particle velocity, the solid phase velocity field is obtained in terms of Lambert $W$ function. Subsequently, this velocity field is substituted in the solid phase continuity equation; transforming it to a semi-linear hyperbolic partial differential equation, which is solved to obtain the solid phase volume fraction field.
\end{abstract}

Keywords Particle-laden flow · Multiphase flow · Eulerian · Hyperbolic partial differential equations · Method of characteristics · Lambert W function

\section{Introduction}

In dilute particle-laden flows, the mathematical models describing the dispersed solid phase can be formulated using either the discrete (Lagrangian) approach or the continuum (Eulerian) approach. In the former, Newton's equations of motion for each particle are solved directly to obtain its position and velocity as function of time; whereas in the latter, the mathematical model of the solid phase is formulated in the hydrodynamic sense so that it describes the average velocity and volume fraction (particle concentration) fields for collections of particles. The most obvious advantage of the Eulerian approach over the Lagranian one is the reduction of the computational costs required to solve the equations of motion for the huge number of particles encountered in realistic multiphase flow problems.
Eulerian models of dilute particle-laden flow, with very low solid volume fraction, are often formulated with the one way coupling assumption [1-3]. For such particle concentrations, inter-particle interactions are rare and consequently, the solid phase momentum equations do not contain the stress tensor term which arises from pressure and viscous forces; thus avoiding closure sub-models used for particle flows with relatively higher concentrations. Furthermore, the effect of particles, for such low concentrations, on both the volume fraction and inter-phase momentum exchange in the fluid phase are negligible. Thus, the only coupling between the two phases arises from the effect of the fluid phase on the particles; due to the hydrodynamics forces, which appear in the momentum equations of the solid phase only. Consequently, the fluid flow field can be obtained by solving its Navier-Stokes equations of a single phase flow model for a given geometry. To solve the flow equations of the particle phase, on the other hand, the fluid

Yahia M. Fouda, Yahia_Fouda@mans.edu.eg| ${ }^{1}$ Department of Mechanical Power Engineering, Faculty of Engineering, Mansoura University, Mansoura 35516, Egypt.

SN Applied Sciences (2019) 1:326 | https://doi.org/10.1007/s42452-019-0338-2

Received: 21 December 2018 / Accepted: 4 March 2019 / Published online: 9 March 2019 
velocity field is used as a known parameter in the particle phase momentum equations.

Previous theoretical studies in Eulerian multiphase flow employing the one way coupling assumption have mainly applied computational approaches to solve the mathematical model $[1,4-6]$. This is probably due to the non-linearity of its hyperbolic governing equations, which prohibits analytical solutions beyond simple flow problems. Nevertheless, analytical solutions, even for simple flow problems, are important in the understanding and validation of such mathematical models, which could become ill-posed in specific flow situations [1]. Thus, the main aim of this paper is to derive an analytical solution of a simple yet unreported Eulerian dilute particle-laden flow problem. This paper presents the analytical solution of a steady two-dimensional flow of dispersed solid particles immersed in a uniform gas velocity field with a linear Stokes-drag coupling the solid phase to the gas.

\section{Governing equations}

The Eulerian governing equations [1-3], in the non-conservative form, of a steady two-dimensional dispersed dilute solid (particle) phase immersed in a uniform gas flow in the Cartesian coordinates, $x$ and $y$, are

$u \frac{\partial \alpha}{\partial x}+v \frac{\partial \alpha}{\partial y}=-\alpha\left(\frac{\partial u}{\partial x}+\frac{\partial v}{\partial y}\right)$

$u \frac{\partial u}{\partial x}+v \frac{\partial u}{\partial y}=\gamma\left(u_{f}-u\right)$

$u \frac{\partial v}{\partial x}+v \frac{\partial v}{\partial y}=\gamma\left(v_{f}-v\right)-g$

where $\alpha$ is the solid phase volume fraction; $u$ and $v$ are the velocity components of the solid phase in $x$ and $y$ directions, respectively; $u_{f}$ and $v_{f}$ are the uniform velocity components of the gas phase in $x$ and $y$ directions, respectively; $g$ is the acceleration due to gravity, which is assumed to act, without loss of generality, along the negative $y$ direction; and $\gamma$ is the reciprocal of the particle relaxation time. The momentum Eqs. (2) and (3) include the drag force as the only interaction term between the two phases. Assuming the flow is within the Stokes regime; which is applicable for low Reynolds number, i.e. $R e \ll 1 ; \gamma$ is constant and given by [1, 2]

$\gamma=18 \mu / \rho_{p} d^{2}$

where $\mu$ is the dynamic viscosity of the gas, $\rho_{p}$ is the particle material density and $d$ is the particle diameter.

Because the momentum Eqs. (2) and (3) do not contain the solid phase volume fraction, $\alpha$, they are uncoupled from the continuity Eq. (1). Thus, the momentum equations should be solved independently to obtain the solid phase velocity field, $u$ and $v$, for a specific inlet velocity boundary condition. Subsequently, the continuity equation, which depends on the velocity field and its derivatives, can be solved to obtain the volume fraction field.

\section{General solution of the momentum equations}

The momentum Eqs. (2) and (3) constitute a system of quasi-linear first order hyperbolic partial differential equations (PDEs). In the matrix form it reads

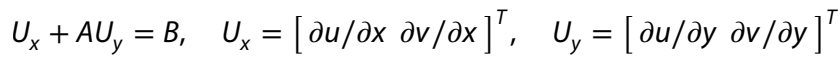

This system is already in the diagonal form because $A$ is the identity matrix multiplied by the system repeated eigenvalue (characteristic), $v / u$. In this paper, the method of characteristics $(\mathrm{MoC})$ is used to solve this system of PDEs analytically. MoC transforms a system of hyperbolic PDEs to a system of ordinary differential equations (ODEs) with a single independent variable in the direction of the hyperbolic PDEs system's characteristics [7]. For the system of hyperbolic PDEs given by Eqs. (2) and (3), the corresponding system of ODEs representing the characteristic equation and compatibility conditions is

$\frac{d x}{d s}=u$

$\frac{d y}{d s}=v$

$\frac{d u}{d s}=\gamma\left(u_{f}-u\right)$

$\frac{d v}{d s}=\gamma\left(v_{f}-v\right)-g$

where $s$ is a dummy variable along the direction of the characteristic curves projected onto the $x y$ plane $[7,8]$.

In order to construct the general solution for a single hyperbolic partial differential equation, a single algebraic equation obtained from two independent first integrals, whose first derivatives along the characteristic direction vanish, should be derived [7]. For the system described by Eqs. (5), (6), (7) and (8), however, the general solution requires the derivation of two independent algebraic equations to obtain the two components of the velocity field, $u$ and $v$. Thus, three independent first integrals; $I_{1}, I_{2}$ and $I_{3}$; ought to be derived from Eqs. (5), (6), (7) and (8). These three independent first integrals can be 
subsequently combined into two algebraic equations to construct the solution. These three independent first integrals, whose first derivatives with respect to the characteristic direction s vanish, are derived in "Appendix A" and can be expressed as

$\mathrm{I}_{1}=\frac{u}{\gamma u_{f}}+\frac{x}{u_{f}}-\frac{v}{\gamma v_{f}-g}-\frac{\gamma y}{\gamma v_{f}-g}$

$\mathrm{I}_{2}=\frac{u}{\gamma u_{f}}+\frac{x}{u_{f}}+\frac{1}{\gamma} \ln \left(u_{f}-u\right)$

$\mathrm{I}_{3}=\ln \left(\frac{u_{f}-u}{v_{f}-g / \gamma-v}\right)$

Accordingly, the general solution of the momentum equations can be expressed as

$\mathrm{I}_{i}=F_{i j}\left(\mathrm{I}_{j}\right), j \neq i$

where $F_{i j}$ is a function to be determined from the initial condition (hereafter referred to as inlet boundary condition).

\section{Uniform inlet solid phase velocity}

In this paper, a uniform solid phase velocity along a straight line parallel to the $y$ direction is used as an inlet boundary condition for the momentum Eqs. (2) and (3). This boundary condition is expressed mathematically as

$u(0, y)=U_{0}, \quad v(0, y)=v_{0}$

where $U_{0}$ and $V_{0}$ are the solid phase inlet velocity in the $x$ and $y$ directions, respectively.

It should be noted that $u=0$ is not an admissible solution for the initial value problem defined by the momentum Eqs. (2) and (3), and the inlet boundary condition (13). This is because for such solution the characteristic curve, whose slope is $v / u$, becomes vertical line, i.e. parallel to the line on which the inlet velocity is prescribed [7, 8], as given by Eq. (13).

Because the momentum equations are hyperbolic, it should be noted that, depending on the curve on which the inlet velocity is prescribed, the solution may not occupy the whole spatial domain. Since the initial conditions were prescribed on the line $y=0$; therefore the solution can either occupy the left $(x>0)$ or right $(x<0)$ half plane, but not both. It is clear that this requires $U_{0}$ and $u_{f}$ being in the same direction, because otherwise the particles will change their direction and the problem becomes ill-posed with $u$ being multi-valued or non-unique. This is different from the Lagrangian model in which any single particle is allowed to change its direction, because time is the only independent variable and the spatial coordinates are dependent variables. A similar situation was reported in the literature where the problem becomes ill-posed when two streams of Eulerian particles intersect [1].

To obtain the first equation describing the general solution, substitute Eqs. (9) and (10) into Eq. (12) as

$\frac{u}{\gamma u_{f}}+\frac{x}{u_{f}}+\frac{1}{\gamma} \ln \left(u_{f}-u\right)=F_{21}\left[\frac{u}{\gamma u_{f}}+\frac{x}{u_{f}}-\frac{v}{\gamma v_{f}-g}-\frac{\gamma y}{\gamma v_{f}-g}\right]$

Then to obtain the particular solution of the momentum Eqs. (2) and (3), substitute the inlet velocity boundary condition given by Eq. (13) into Eq. (14) as

$\frac{U_{0}}{\gamma u_{f}}+\frac{1}{\gamma} \ln \left(u_{f}-U_{0}\right)=F_{21}\left[\frac{U_{0}}{\gamma u_{f}}-\frac{v_{0}}{\gamma v_{f}-g}-\frac{\gamma y}{\gamma v_{f}-g}\right]$

which shows that the function $F_{21}$ is constant for any arbitrary argument $\vartheta$ given by

$F_{21}(\vartheta)=\frac{U_{0}}{\gamma u_{f}}+\frac{1}{\gamma} \ln \left(u_{f}-U_{0}\right)$

Then substitute $F_{21}(\vartheta)$, given by Eq. (16), into Eq. (14) as

$\frac{u}{\gamma u_{f}}+\frac{x}{u_{f}}+\frac{1}{\gamma} \ln \left(u_{f}-u\right)=\frac{U_{0}}{\gamma u_{f}}+\frac{1}{\gamma} \ln \left(u_{f}-U_{0}\right)$

which is a non-linear algebraic equation in a single variable $u$. After some manipulation, Eq. (17) can be written as

$$
\begin{aligned}
\left(\frac{u-u_{f}}{u_{f}}\right) \exp \left(\frac{u-u_{f}}{u_{f}}\right)= & \left(\frac{U_{0}-u_{f}}{u_{f}}\right) \exp \left(\frac{U_{0}-u_{f}}{u_{f}}\right) \\
& \times \exp \left(\frac{-\gamma x}{u_{f}}\right)
\end{aligned}
$$

which is a transcendental algebraic equation in the variable $\left(u-u_{f}\right) / u_{f}$, whose solution is the Lambert $W$ function $[9,10], W(z)$, with $z$ being the function argument, as

$\left(\frac{u-u_{f}}{u_{f}}\right)=W\left(\left(\frac{U_{0}-u_{f}}{u_{f}}\right) \exp \left(\frac{U_{0}-u_{f}}{u_{f}}\right) \exp \left(\frac{-\gamma x}{u_{f}}\right)\right)$

An explicit solution of the velocity component in the $x$ direction, $u$, can be written as

$u=u_{f}[1+W(f(x))]$

$f(x)=\left(\frac{U_{0}-u_{f}}{u_{f}}\right) \exp \left(\frac{U_{0}-u_{f}}{u_{f}}\right) \exp \left(\frac{-\gamma x}{u_{f}}\right)$

Equation (20) describes the decay of the slip velocity component in $x$ direction, $u-u_{f}$. As $x$ increases to approach infinity; $f(x)$, given by Eq. (21), approaches zero. 
In order to be consistent with the boundary condition given by Eq. (13), it should be noted that in addition to being in the same direction as the inlet solid phase velocity component in $x$ direction, $U_{0}$, the gas phase velocity component in $x$ direction, $u_{f}$, should not be zero; because otherwise the characteristics, as $x$ approaches infinity, become parallel to the curve on which the initial data is prescribed [7]. Note that for the well-posed condition which requires $U_{0}$ and $u_{f}$ to be in the same direction, the value of $\mathrm{W}(f(x))$ is always greater than -1 ; and thus, the solution given by Eq. (20) refers to the upper branch of Lambert $\mathrm{W}$ function only.

Now, to obtain the general solution of $v$, the two independent first integrals, $I_{1}$ and $l_{3}$, defined by Eqs. (9) and (11), respectively, are combined as

$\ln \left(\frac{u_{f}-u}{v_{f}-g / \gamma-v}\right)=F_{31}\left[\frac{u}{\gamma u_{f}}+\frac{x}{u_{f}}-\frac{v}{\gamma v_{f}-g}-\frac{\gamma y}{\gamma v_{f}-g}\right]$

By substituting the inlet velocity boundary condition, given by Eq. (13), into Eq. (22), it is clear that the function $F_{31}$ is constant for any arbitrary argument $\vartheta$ as

$F_{31}(\vartheta)=\ln \left(\frac{u_{f}-U_{0}}{v_{f}-g / \gamma-V_{0}}\right)$

Then substituting $F_{31}(\vartheta)$, given by Eq. (23), into Eq. (14), yields

$\frac{u_{f}-u}{v_{f}-g / \gamma-v}=\frac{u_{f}-U_{0}}{v_{f}-g / \gamma-V_{0}}$

which results in an explicit expression for the velocity component $v$ as a function of the velocity component $u$ given by

$v=\left(v_{f}-g / \gamma\right)-\left(v_{f}-g / \gamma-v_{0}\right)\left(\frac{u_{f}-u}{u_{f}-U_{0}}\right)$

Substituting the expression of $u$, as given by Eq. (20), into Eq. (25), gives the following explicit relation for the velocity component in the $y$ direction, $v$, as a function of $x$ coordinate

$v=\left(v_{f}-g / \gamma\right)-\frac{u_{f}\left(v_{f}-g / \gamma-v_{0}\right)}{\left(U_{0}-u_{f}\right)} W(f(x))$

Equation (26) shows that the particle velocity component $v$ approaches the terminal velocity $\left(v_{f}-g / \gamma\right)$ simultaneously with the velocity component $u$, when $f(x)$ approaches zero and $x$ approaches infinity. The decay in the slip velocity in $y$ direction $\left(v-v_{f}+g / \gamma\right)$ is a function of $\mathrm{W}(f(x))$ and follows the same trend as $u$.

For the case of $U_{0}=u_{f}$; the velocity component in $x$ direction, $u$, is already fully developed, i.e. $u=u_{f}$. Thus, the velocity component in $y$ direction, $v$, obtained using Eq. (26), becomes undetermined quantity. This can be avoided by eliminating $\left(U_{0}-u_{f}\right)$ and $W(f(x))$ from Eq. (26). Using the identity of Lambert $W$ function with any arbitrary argument $z$ [9]

$\mathrm{W}(z)=z \exp (-\mathrm{W}(z))$

it can be shown that the velocity component $v$ in the case of $U_{0}=u_{f}$ is

$v=\left(v_{f}-g / \gamma\right)-\left(v_{f}-g / \gamma-v_{0}\right) \exp \left(\frac{-\gamma X}{u_{f}}\right)$

which shows that the slip velocity in $y$ direction, $\left(v-v_{f}+g / \gamma\right)$, decays exponentially so that the particle velocity component $v$ becomes fully developed with the terminal velocity in $y$ direction $\left(v_{f}-g / \gamma\right)$ as $x$ approaches infinity. Figure 1 shows the decay of the dimensionless velocity in $y$ direction, $v /\left(v_{f}-g / \gamma\right)$, with the dimensionless position, $\gamma x / u_{f}$; for different values of the dimensionless parameter, $v_{0} /\left(v_{f}-g / \gamma\right)$.

Now after the obtaining the velocity field, the characteristic curves can be determined by solving the characteristic $\mathrm{ODE}, d y / d x=v / u$. In the case of $u_{f} \neq U_{0}$, this characteristic ODE is given by

$\frac{d y}{d x}=\frac{v}{u}=b\left[\frac{1+a \mathrm{~W}(f(x))}{1+\mathrm{W}(f(x))}\right]$

where the parameters $a$ and $b$ are defined as

$a=\frac{u_{f}\left(v_{f}-g / \gamma-v_{0}\right)}{\left(u_{f}-U_{0}\right)\left(v_{f}-g / \gamma\right)}, \quad b=\frac{\left(v_{f}-g / \gamma\right)}{u_{f}}$

Re-arranging Eq. (29) gives

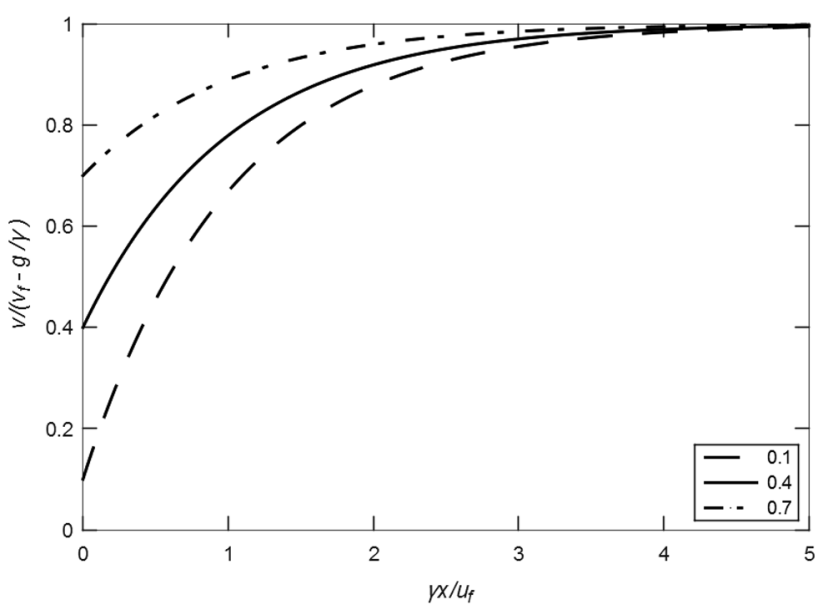

Fig. 1 Effect of $v_{0} /\left(v_{f}-g / \gamma\right)$ on the decay of the dimensionless velocity, $v /\left(v_{f}-g / \gamma\right), u_{0}=u_{f}$ 
$\frac{d y}{d x}=b a+\frac{b(1-a)}{1+W(f(x))}$

Equation (31) is solved analytically using the separation of variables method as shown in "Appendix B". Its solution results in the following algebraic equation describing a family of characteristic curves

$$
\begin{aligned}
y= & \left(\frac{v_{f}-g / \gamma}{u_{f}}\right) x+\frac{1}{\gamma}\left(\frac{U_{0}}{u_{f}}\left(v_{f}-g / \gamma\right)-v_{0}\right) \\
& \times\left(\frac{W(f(x))}{\left(U_{0}-u_{f}\right) / u_{f}}-1\right)+y_{0}
\end{aligned}
$$

where the constant of integration, $y_{0}$, represents the intercept of the $y$ axis for each characteristic curve.

In the case of $U_{0}=u_{f}=u$, Eq. (28) is used to express the velocity component in the $y$ direction, $v$, resulting in the following characteristic ODE

$\frac{d y}{d x}=\frac{v}{u}=\frac{1}{u_{f}}\left[\left(v_{f}-g / \gamma\right)-\left(v_{f}-g / \gamma-v_{0}\right) \exp \left(\frac{-\gamma x}{u_{f}}\right)\right]$,

whose solution using the separation of variables method is

$y=\left(\frac{v_{f}-g / \gamma}{u_{f}}\right) x+\frac{1}{\gamma}\left(v_{f}-g / \gamma-v_{0}\right) \exp \left(\frac{-\gamma x}{u_{f}}\right)+y_{0}$

By definition, the characteristic curves of the momentum Eqs. (2) and (3) are the streamlines of the solid phase. For both cases of characteristic curves shown in Eqs. (32) and (34), the first term on the right-hand side describes a straight line. The second term on the right-hand side is, however, an equation of a curve which is either Lambert $W$ function in the case of $u_{f} \neq U_{0}$, or exponential in the case of $U_{0}=u_{f}=u$. For large values of $x$, the curved parts of the characteristic curves vanish and the particle phase streamlines are straight lines whose slope equals that of fully developed solid phase flow with the particle terminal velocity. Figures 2 and 3 show the relaxation of the particle phase characteristics, obtained from Eq. (32) and (34), respectively; to the fluid streamlines.

\section{Solution of the continuity equation}

Having obtained the particular solution of the momentum equations for a uniform inlet solid phase velocity defined by Eq. (13), the velocity field $(u, v)$ is substituted into the continuity Eq. (1) in order to obtain the analytical solution of the volume fraction field, $\alpha$. The second term on the right-hand side of the continuity Eq. (1), $-\alpha(\partial v / \partial y)$, vanishes; because the velocity component $v$ is a function of the $x$ coordinate only as shown in Eqs. (26) and (28). Thus, the continuity equation can be written as

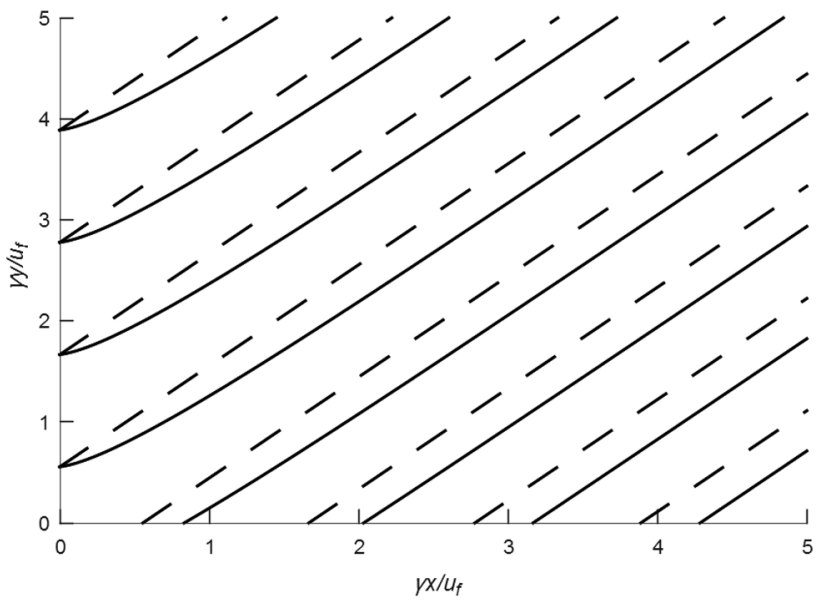

Fig. 2 The relaxation of particle phase characteristics for $u_{f} \neq U_{0}$ (solid lines) to the fluid streamlines (dashed lines)

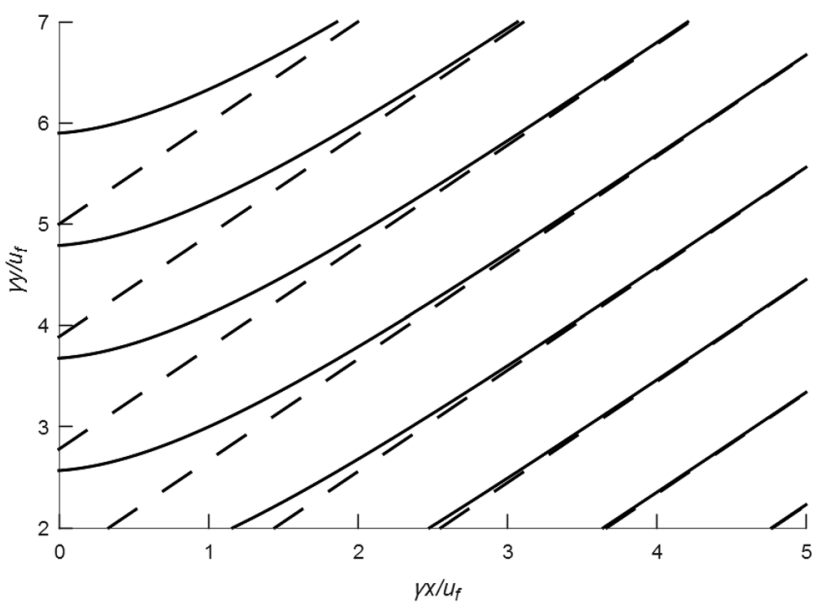

Fig. 3 The relaxation of particle phase characteristics for $U_{0}=u_{f}=u$ (solid lines) to the fluid streamlines (dashed lines)

$u \frac{\partial \alpha}{\partial x}+v \frac{\partial \alpha}{\partial y}=-\alpha \frac{\partial u}{\partial x}$

Using the calculus properties of Lambert $\mathrm{W}$ function [9], and the chain rule; the first derivative of the velocity component $u$ with respect to $x$ is

$\frac{\partial u}{\partial x}=\frac{-\gamma \mathrm{W}(f(x))}{1+\mathrm{W}(f(x))}$

Now, the continuity equation is a semi-linear hyperbolic PDE whose characteristics are the same as the momentum Eqs. (2) and (3), i.e. $v / u$. To solve this continuity equation analytically, the method of characteristics (MoC) is used again. A non-linear transformation is applied to the independent variables $(x, y)$ resulting in a new set of independent variables $(x, \eta)$. Here $\eta$ is chosen to be in the direction of the characteristic curves, for either $u_{f} \neq U_{0}$ or $u_{f}=U_{0}$, as 
$\eta=\left\{\begin{array}{l}y-\left(\frac{v_{f}-g / \gamma}{u_{f}}\right) x-\frac{1}{\gamma}\left(\frac{U_{0}}{u_{f}}\left(v_{f}-g / \gamma\right)-v_{0}\right)\left(\frac{w(f(x))}{\left(U_{0}-u_{f}\right) / u_{f}}-1\right), \quad U_{0} \neq u_{f} \\ y-\left(\frac{v_{f}-g / \gamma}{u_{f}}\right) x-\frac{1}{\gamma}\left(v_{f}-g / \gamma-v_{0}\right) \exp \left(\frac{-\gamma x}{u_{f}}\right), \quad U_{0}=u_{f}\end{array}\right.$

Note that the transformation defined by Eq. (37) is oneto-one because its Jacobian equals unity, i.e. does not vanish $[7,8]$. Using the chain rule to express $\alpha$ as a function of $(x, \eta)$ yields

$\frac{\partial \alpha}{\partial x}=\left(\frac{\partial \alpha}{\partial x}\right)+\left(\frac{\partial \alpha}{\partial \eta}\right)\left(\frac{\partial \eta}{\partial x}\right), \frac{\partial \alpha}{\partial y}=\left(\frac{\partial \alpha}{\partial \eta}\right)\left(\frac{\partial \eta}{\partial y}\right)$

Substituting Eq. (38) into the continuity Eq. (35), in case $u \neq 0$, gives

$\frac{\partial \alpha}{\partial x}+\frac{\partial \alpha}{\partial \eta}\left[\frac{\partial \eta}{\partial x}+\left(\frac{v}{u}\right) \frac{\partial \eta}{\partial y}\right]+\left(\frac{\alpha}{u}\right) \frac{\partial u}{\partial x}=0$

Since $\eta$ is in the direction of the characteristic curve, the second term on the left-hand side of Eq. (39) vanishes. Consequently, the velocity component $u$ is the only parameter required to solve the continuity equation. By substituting the expression of $\partial u / \partial x$ from Eq. (36) and $u$ from Eq. (20), the continuity equation is

$\frac{\partial \alpha}{\partial x}-\frac{\gamma \mathrm{W}(f(x))}{u_{f}(1+\mathrm{W}(f(x)))^{2}} \alpha=0$

which is a linear first order PDE in one independent variable $\alpha$. Thus, it can be solved in a similar way as an ODE. Using the integrating factor method [7], Eq. (40) can be written as

$\left(\exp \left[\int \beta(x) d x\right]\right) \frac{\partial \alpha}{\partial x}+\alpha\left(\exp \left[\int \beta(x) d x\right]\right) \beta(x)=0$

with the function $\beta(x)$ defined as

$\beta(x)=\frac{-\gamma \mathrm{W}(f(x))}{u_{f}(1+\mathrm{W}(f(x)))^{2}}$

Thus, the continuity equation can be expressed as

$\frac{\partial}{\partial x}\left(\alpha \exp \left[\int \beta(x) d x\right]\right)=0$

As shown "Appendix $C$ ", the integral $\int \beta(x) d x$ is evaluated as

$\int \beta(x) d x=\ln (1+\mathrm{W}(f(x)))$

which results in the continuity equation being $\frac{\partial}{\partial x}[(1+\mathrm{W}(f(x))) \alpha]=0$,

whose general solution is

$\alpha(x, \eta)=\frac{G(\eta)}{1+\mathrm{W}(f(x))}$

where $G$ is an arbitrary function to be determined from the inlet boundary condition. Applying an inverse transformation from the new coordinates $(x, \eta)$ to the original coordinates $(x, y)$, the general solution of the volume fraction field, $\alpha$, is

$\alpha(x, y)=\frac{G(\eta(x, y))}{1+W(f(x))}=\left(\frac{u_{f}}{u(x)}\right) G(\eta(x, y))$

The inlet boundary condition of the continuity equation ought to be prescribed on the same curve as that of the momentum equations, because the volume fraction field cannot have a different domain from that of the velocity field. Assuming that the inlet boundary condition of the volume fraction takes the form

$\alpha(0, y)=\alpha_{0} f(y)$

where $f(y)$ is an arbitrary function of the independent variable $y$, which describes the inlet volume fraction distribution along the line $x=0$. The function $G(\eta(x=0, y))$ is expressed as

$G(\eta(x=0, y))=G(y)=\alpha_{0} \frac{U_{0}}{u_{f}} f(y)$

which solves the volume fraction field, $\alpha$, as

$\alpha(x, y)=\alpha_{0}\left(\frac{U_{0}}{u(x)}\right) f(y)$

which shows that the mass flux in the $x$ direction is constant along the lines of $y$ equals constant.

In the case of $u_{f}=U_{0}$, the volume fraction is a function of the $y$ coordinate only as

$\alpha(x, y)=\alpha_{0}\left(\frac{U_{0}}{u_{f}}\right) f(y)$

In the case of uniform inlet volume fraction, $f(y)=1$, the volume fraction depends on the $x$ coordinate only as

$\alpha(x, y)=\alpha(x)=\alpha_{0} \frac{U_{0}}{u(x)}$ 


\section{Conclusions}

The analytical solution of the momentum equations showed that the decay in the solid phase slip velocity has the form of Lambert $W$ function when $u_{f} \neq U_{0}$, and is exponential when $u_{f}=U_{0}$. The analytical solution of the continuity equation showed that the solid volume fraction field multiplied by the velocity component $u$ is constant along the $x$ direction; and its variation along the $y$ direction depends on its inlet boundary condition.

\section{Compliance with ethical standards}

Conflict of interest The author declares that there is no conflict of interest.

\section{Appendix A: Derivation of independent first integrals}

The independent first integrals of Eqs. (5), (6), (7) and (8), whose first derivatives along the characteristic direction $s$ vanish, are derived as follows:

Dividing Eq. (7) by $\gamma u_{f}$ gives

$\frac{d}{d s}\left(\frac{u}{\gamma u_{f}}\right)=1-\frac{u}{u_{f}}$

Dividing Eq. (5) by $u_{f}$ gives

$\frac{d}{d s}\left(\frac{x}{u_{f}}\right)=\frac{u}{u_{f}}$

Adding equations (53) and (54) gives

$\frac{d}{d s}\left(\frac{u}{\gamma u_{f}}+\frac{x}{u_{f}}\right)=1$

Dividing Eq. (8) by $\left(\gamma v_{f}-g\right)$ gives

$\frac{d}{d s}\left(\frac{v}{\gamma v_{f}-g}\right)=1-\frac{\gamma v}{\gamma v_{f}-g}$

Multiplying Eq. (6) by $\gamma /\left(\gamma v_{f}-g\right)$ gives

$\frac{d}{d s}\left(\frac{\gamma y}{\gamma v_{f}-g}\right)=\frac{\gamma v}{\gamma v_{f}-g}$

Adding equations (56) and (57) gives

$\frac{d}{d s}\left(\frac{v}{\gamma v_{f}-g}+\frac{\gamma y}{\gamma v_{f}-g}\right)=1$

Now, subtracting equation (58) from equation (55) gives

$\frac{d}{d s}\left(\frac{u}{\gamma u_{f}}+\frac{x}{u_{f}}-\frac{v}{\gamma v_{f}-g}-\frac{\gamma y}{\gamma v_{f}-g}\right)=0$, which is the equation for one of the independent first integrals, $l_{1}$, as shown in Eq. (9).

Now to derive an equation for the second independent first integral, $I_{2}$, we use the first derivative of the logarithm of the right-hand side of Eq. (7), which is

$\frac{d}{d s}\left(\ln \left(u_{f}-u\right)\right)=\frac{-d u / d s}{\left(u_{f}-u\right)}$

Substituting the expression of $d u / d s$ from Eq. (7) into equation (60) and dividing by $\gamma$ gives

$\frac{d}{d s}\left(\frac{1}{\gamma} \ln \left(u_{f}-u\right)\right)=-1$

Then adding equation (61) to equation (55) gives

$\frac{d}{d s}\left(\frac{u}{\gamma u_{f}}+\frac{x}{u_{f}}+\frac{1}{\gamma} \ln \left(u_{f}-u\right)\right)=0$,

which is the equation for the second independent first integral, $I_{2}$, as shown in Eq. (10).

Now to derive the equation for the third independent first integral, $l_{3}$, we use the first derivative of the logarithm of the right-hand side of Eq. (8) and divide by $\gamma$, which is

$\frac{d}{d s}\left(\frac{1}{\gamma} \ln \left(v_{f}-v-g / \gamma\right)\right)=-1$

Then subtracting equation (63) from equation (61) gives

$\frac{d}{d s}\left(\ln \left(\frac{u_{f}-u}{v_{f}-g / \gamma-v}\right)\right)=0$,

which is the equation for the third independent first integral, $l_{3}$, as shown in Eq. (11).

\section{Appendix B: Solution of the characteristic ODE for $u_{f} \neq U_{0}$}

Integrating the characteristic Eq. (31) gives

$y=b a x+b(1-a) \int \frac{d x}{1+W(f(x))}+C_{1}$

where $C_{1}$ is the constant of integration. The integral $I=\int d x /(1+W(f(x))$ is evaluated as follows:

Defining a non-linear transformation of the independent variable as

$t=f(x)=C \exp \left(\frac{-\gamma x}{u_{f}}\right), \quad C=\left(\frac{U_{0}-u_{f}}{u_{f}}\right) \exp \left(\frac{U_{0}-u_{f}}{u_{f}}\right)$

The derivatives of the transformation are 
$d t=\frac{-\gamma}{u_{f}} t d x, \quad d x=\frac{-u_{f} d t}{\gamma t}$

Applying the transformation defined by equations (66) and (67) to the integral / gives

$I=\frac{-u_{f}}{\gamma} \int \frac{d t}{t(1+W(t))}$

Now define another non-linear transformation as

$Z=(1+\mathrm{W}(t))$

An explicit relation of the variable $t$ as a function of the variable $Z$ is

$W(t)=Z-1, \quad t=(Z-1) \exp (Z-1)$

The derivative of the transformation is

$d t=Z \exp (Z-1) d Z$

Applying the transformation to the integral / gives

$$
\begin{aligned}
I & =\frac{-u_{f}}{\gamma} \int \frac{d Z}{(Z-1)}=\frac{-u_{f}}{\gamma} \ln (Z-1)=\frac{-u_{f}}{\gamma} \ln (\mathrm{W}(t)) \\
& =\frac{-u_{f}}{\gamma} \ln (\mathrm{W}(f(x)))
\end{aligned}
$$

Substituting the expression of $I$, as given by equation (72), in the characteristic equation (65)

$y=b a x+b(1-a)\left(\frac{-u_{f}}{\gamma} \ln (W(f(x)))\right)+C_{1}$

To find $C_{1}$, set $x=0$ and $y=0$ for the characteristic line passing through the origin, which gives

$C_{1}=\frac{u_{f} b(1-a)}{\gamma} \ln (\mathrm{W}(C))$

Then substitute equation (74) into equation (73) as

$y=b a x-\frac{u_{f} b(1-a)}{\gamma}[\ln (\mathrm{W}(f(x)))-\ln (\mathrm{W}(C))]$

Using the identity of Lambert W function [9]

$\ln (\mathrm{W}(Z))=\ln (Z)-\mathrm{W}(Z)$

It can be shown that the logarithmic terms in the algebraic equation describing the characteristic curves (B.9) is

$\ln (\mathrm{W}(f(x)))-\ln (\mathrm{W}(C))=\ln \left(\frac{f(x)}{C}\right)-[\mathrm{W}(f(x))-\mathrm{W}(C)]$

Substitute the expression of $f(x)$ from equation (66) into equation (75) as

$y=b x+\frac{u_{f} b(1-a)}{\gamma}[W(f(x))-W(C)]$
From the definition of Lambert $\mathrm{W}$ function [9] and equation (66), it than be shown that

$\mathrm{W}(C)=\mathrm{W}\left(\left(\frac{U_{0}-u_{f}}{u_{f}}\right) \exp \left(\frac{U_{0}-u_{f}}{u_{f}}\right)\right)=\left(\frac{U_{0}-u_{f}}{u_{f}}\right)$

Using Eq. (30), it can be shown that

$b(1-a)=\frac{\left(v_{f}-g / \gamma\right)\left(u_{f}-U_{0}\right)-u_{f}\left(v_{f}-g / \gamma-v_{0}\right)}{u_{f}\left(u_{f}-U_{0}\right)}$

Substituting equations (79) and (80) into equation (78), it can be shown that the equation of the characteristic curve passing through the origin is

$y=\left(\frac{v_{f}-g / \gamma}{u_{f}}\right) x+\frac{1}{\gamma}\left(\frac{U_{0}}{u_{f}}\left(v_{f}-g / \gamma\right)-v_{0}\right)\left(\frac{W(f(x))}{\left(U_{0}-u_{f}\right) / u_{f}}-1\right)$

An arbitrary value of the $y$ axis intercept, $y_{0}$, can be added to equation (81), as shown in Eq. (32), to define the equations describing a family of parallel characteristic curves.

\section{Appendix C: Evaluation of the integral $\int \beta(x) d x$}

The integral $\int \beta(x) d x$ is given by Eq. (42) as

$\int \beta(x) d x=\int \frac{-\gamma \mathrm{W}(f(x))}{u_{f}(1+\mathrm{W}(f(x)))^{2}} d x$

Applying the transformation defined by equations (66) and (67) to the integral $\int \beta(x) d x$ gives

$\int \beta(x) d x=\int \frac{-\gamma \mathrm{W}(f(x))}{u_{f}(1+\mathrm{W}(f(x)))^{2}} d x=\int \frac{\mathrm{W}(t)}{t(1+\mathrm{W}(t))^{2}} d t$

Applying another transformation; defined by equations (69), (70) and (71); to the integral $\int \beta(x) d x$ and substituting with the original variable, $x$, gives

$\int \beta(x) d x=\int \frac{d Z}{Z}=\ln Z=\ln (1+W(t))=\ln (1+W(f(x)))$

\section{References}

1. Slater SA, Young JB (2001) The calculation of inertial particle transport in dilute gas-particle flows. Int J Multiph Flow 27:61-87. https://doi.org/10.1016/S0301-9322(99)00122-6

2. Foster MR, Duck PW, Hewitt RE (2006) Boundary layers in a dilute particle suspension. Proc R Soc Lond Ser A 462:1145. https://doi.org/10.1098/rspa.2005.1611 
3. Fernandez de la Mora J (1982) Two dimensional stagnation point flow of a dusty gas near an oscillating plate. Acta Mech 43:261-265. https://doi.org/10.1007/bf01176286

4. Sachdev JS, Groth CPT, Gottlieb JJ (2007) Numerical solution scheme for inert, disperse, and dilute gas-particle flows. Int J Multiph Flow 33:282-299. https://doi.org/10.1016/j.ijmultipha seflow.2006.09.001

5. Slater SA, Leeming AD, Young JB (2003) Particle deposition from two-dimensional turbulent gas flows. Int J Multiph Flow 29:721-750. https://doi.org/10.1016/S0301 -9322(03)00037-5

6. Wu Z, Young JB (2012) The deposition of small particles from a turbulent air flow in a curved duct. Int J Multiph Flow 44:3447. https://doi.org/10.1016/j.ijmultiphaseflow.2012.03.011
7. Kersalé E (2018) Analytic solutions of partial differential equations. http://www1.maths.leeds.ac.uk/ kersale/Teach/M3414 /Notes/m3414_1.pdf. Accessed 5 Apr 2018

8. John F (1978) The single first-order equation. In: John F (ed) Partial differential equations. Springer, New York, pp 1-30

9. Corless RM, Gonnet GH, Hare DEG, Jeffrey DJ, Knuth DE (1996) On the Lambert W function. Adv Comput Math 5:329-359. https ://doi.org/10.1007/BF02124750

10. Hu H, Zhao YP, Guo YJ, Zheng MY (2012) Analysis of linear resisted projectile motion using the Lambert $\mathrm{W}$ function. Acta Mech 223:441-447. https://doi.org/10.1007/s00707-011-0571-2

Publisher's Note Springer Nature remains neutral with regard to jurisdictional claims in published maps and institutional affiliations. 